\title{
Linx
}

Revue des linguistes de l'université Paris X Nanterre

$12 \mid 2002$

"Comme la lettre dit la vie »

\section{Le chevalier - la chevaleresse : Le développement d'un suffixe spécifique pour les « estats » féminins au Moyen Age}

Sabine Lehmann

\section{OpenEdition}

Journals

Édition électronique

URL : http://journals.openedition.org/linx/1291

DOI : $10.4000 /$ linx. 1291

ISSN : 2118-9692

Éditeur

Presses universitaires de Paris Nanterre

Édition imprimée

Date de publication : 1 octobre 2002

Pagination : 125-131

ISSN : 0246-8743

Référence électronique

Sabine Lehmann, «Le chevalier - la chevaleresse : Le développement d'un suffixe spécifique pour les " estats » féminins au Moyen Age », Linx [En ligne], 12 | 2002, mis en ligne le 10 octobre 2012, consulté le 10 décembre 2020. URL : http://journals.openedition.org/linx/1291 ; DOI : https://doi.org/10.4000/ $\operatorname{lin} x .1291$

Ce document a été généré automatiquement le 10 décembre 2020.

Département de Sciences du langage, Université Paris Ouest 


\title{
Le chevalier - la chevaleresse : Le développement d'un suffixe spécifique pour les " estats » féminins au Moyen Age
}

\author{
Sabine Lehmann
}

1 La commodité d'un suffixe pour marquer l'appartenance à une catégorie ou à un groupe dans le cadre d'un classement est évidente. Pensons, par exemple, au classement des êtres humains sur le plan fonctionnel. Les langues européennes disposent de suffixes d'agent qui s'ajoutent aux radicaux spécifiant les diverses activités exercées par cet agent.

Dans la tradition du latin et des langues romanes, ce système a bien fonctionné pour nommer des groupes de professions à définition économique étroite. Nous voudrions citer quelques exemples de désignations de l'être humain choisies selon le critère de l'appartenance à un groupe professionnel, relevés dans les ballades d'Eustache Deschamps (1344-1404). L'œuvre de ce poète, représentant de la petite noblesse de robe, témoigne de la grande mobilité sociale caractérisant la période des $14^{\mathrm{e}}-15^{\mathrm{e}}$ siècles. Dans ses ballades, le lecteur rencontre des représentants différents de l'artisanat (peletiers, ballade 906 , v. 16 ; savetier, ballade 908, v. 3 ; ballade 920 : cousturier, v. 15, sellier, v. 16, lormiers, v. 21, heaumiers, v. 24, brodeur, v. 12), ainsi que d'activités liées au monde rural ( barbier de bos, ballade 910, v. 7), au service (bouteillier, ballade 869, v. 9 ; despensier, ballade 869, v. 1), au domaine du savoir (astronomien, ballade 833, v. 6 ; naturien, ballade 891, v. 1 ; fisicien, ballade 833, v. 15 ; surgien, ballade 902, v. 5) ou de l'art (soufleur, ballade 923, v. 9) ${ }^{1}$.

3 Le grand nombre d'attestations nous prouve que ce système de fonctionnement (adjonction d'un suffixe d'agent - ier, -ien, -eur à une base désignant l'objet ou le contenu de l'activité) a été très productif au Moyen Age et reflète l'évolution des structures sociales $^{2}$. Mais il ne s'est guère étendu aux formulations d'un système global groupant toute la société en un très petit nombre de catégories - selon le fonctionnement de la société en trois ordres ou trois états. 


\section{Les « estats du monde »}

4 Dans une première partie, nous nous proposons d'analyser l'évolution d'un suffixe spécifique pour la désignation des « estats » de la société médiévale.

5 La Chrétienté médiévale s'est souvent représentée en schémas binaires, en couples antithétiques - le plus général et le plus important a été l'opposition clercs / laïcs. Mais à côté du critère de la religion, la puissance, elle aussi, a été une ligne de clivage importante. Dans le haut Moyen Age, elle s'est traduite par l'opposition potens / pauper, remplacée à partir du $13^{\mathrm{e}}$ siècle par le couple riche / pauvre, ce qui traduisait les progrès de l'économie monétaire et la promotion de la richesse comme source ou conséquence de la puissance.

6 Pourtant, comme le souligne Jacques Le Goff, «la prise de conscience d'une complexification de la société a conduit les hommes du Moyen Age à préférer des schémas plus articulés que le simpliste schéma binaire $»^{3}$. Un de ces principaux schémas fut celui qui introduisit tout simplement une catégorie intermédiaire, celle des moyens entre les catégories extrêmes des grands et des petits: majores, mediocres, minores. Ce schéma fut particulièrement pertinent au début du $13^{\mathrm{e}}$ siècle, quand les bourgeois des villes apparurent comme des mediocres entre l'aristocratie des grands laïcs et ecclésiastiques et la masse des paysans et citadins sans importance.

7 Mais le schéma qui rencontra le plus de succès, chez les clercs du Moyen Age et chez les historiens de l'époque contemporaine, est le schéma tri-fonctionnel reconnu par Georges Dumézil comme une structure fondamentale de la culture indo-européenne ${ }^{4}$. Ce schéma apparaît dans l'occident chrétien au $9^{\mathrm{e}}-10^{\mathrm{e}}$ siècle et s'impose à partir d'un texte de l'évêque Adalbéron de Laon dans son Poème au roi Robert (le Pieux), vers 1030. Adalbéron distingue trois composantes dans la société chrétienne: oratores, bellatores, laboratores, ceux qui prient, ceux qui combattent, ceux qui travaillent. Le schéma tri-fonctionnel correspond au paysage social des lendemains de l'An mille. Il donne l'image d'une société en apparence harmonieuse et complémentaire. Mais, en réalité, cette tripartition de la société renforce l'inégalité sociale entre les trois groupes. Malgré l'apparition de clivages sociaux et l'évolution des structures sociales, on parle, depuis les travaux de Georges Duby et de Jacques Le Goff ${ }^{5}$, à propos de la société du $12^{\mathrm{e}}, 13^{\mathrm{e}}$ et même encore du $15^{\mathrm{e}}$ siècle, d'oratores, bellatores et laboratores, sans se rendre compte qu'à ces époques, ces termes ne faisaient plus partie de l'usage linguistique.

8 La série ternaire en -atores ressemble plus à une nomenclature scientifique à valeur abstraite qu'à un ensemble de termes désignant des représentants concrets des différents groupes sociaux. En effet, dans son étude "Suffixes et taxinomie sociale: le cas des 'trois ordres' ", Jean Batany montre que dans le latin chrétien, le suffixe -tor contribuait à structurer le lexique à partir d'une idéologie de la responsabilité. Les mots en -tor sont utilisés comme des «projets idéologiques purs, envisagés en-deçà de leurs applications précises $»^{6}$. Appliqué à la société globale, le suffixe d'agent prend donc une valeur abstraite qui s'oppose à son fonctionnement concret dans le domaine de la désignation des métiers manuels.

Contrairement à l'unité du système latin, avec sa formule en -atores, on découvre dans des textes de langue française $\mathrm{du} 12^{\mathrm{e}}$ et $13^{\mathrm{e}}$ siècles une dispersion morphologique étonnante des désignations. Jean Batany a relevé chez Etienne de Fougères: clerc, 
chevalier, païsant ; chez Benoît : chevaliers, clercs, vilains; chez Hugues de Berzé : provoires, chevaliers, laboreours; chez le Reclus de Molliens : cler, chevalier, laborier ${ }^{7}$. Ces exemples nous montrent que les suffixes sont peu employés pour opposer les catégories sociales: on a seulement chevalieret laborier dans la dernière série d'attestations. Nous sommes loin du système que forment les termes en -atores de l'An mille. Le seul terme constant d'un texte à l'autre est chevalier, qui fonctionne comme un terme de base autour duquel se constitue le schéma. Celui qui protège les deux autres ordres par les armes, le représentant de cette nouvelle couche sociale de combattants à cheval, trouve son existence confirmée par l'expression linguistique la plus stable. Cette nouvelle couche sociale va devenir une nouvelle noblesse ${ }^{8}$, la chevalerie.

L'utilisation du terme chevalerie s'inscrit dans la grande tendance, se manifestant à partir $\mathrm{du} 13^{\mathrm{e}}$ siècle, à utiliser un suffixe abstrait, généralement -ie, pour la désignation des catégories du schéma ternaire. C'est ce terme même qui a imposé la prédominance du suffixe -ie, mettant en avant une valeur abstraite, inanimée, attribuée à une catégorie de personnes appartenant au même niveau social, à la place d'un pluriel d'une désignation de personne.

11 A la fin $\mathrm{du} 14^{\mathrm{e}}$, tout au début du $15^{\mathrm{e}}$ siècle, une tentative d'homogénéisation morphologique est faite par Christine de Pizan qui dans le Chemin de long estude (1402/03) ${ }^{9}$ aligne trois éléments en -esse : Sagesse, Noblesse, Richesse. Mais, le terme de chevalerie étant trop bien établi dans l'usage et désignant une catégorie centrale du paysage social du Moyen Age, la poétesse se sent obligée de l'ajouter à la série en -esse, s'éloignant ainsi du système des « trois états ».

12 Nous rencontrons le terme de chevalerie également dans l'univers poétique de Rutebeuf, ce poète du $13^{\mathrm{e}}$ siècle pour lequel cette catégorie sociale a perdu son statut prestigieux et n'est plus comparable à la chevalerie des premières chansons de geste. Dans les Plaies $d u$ $M_{0 n d} e^{10}$, Rutebeuf étend ses critiques quant à la situation de décadence dans laquelle se trouve la chevalerie, aux deux autres catégories désignées par un syntagme comportant le collectif gent et un adjectif spécifiant la nature de ce groupe selon son appartenance à l'état ecclésiastique ou son statut laïque : gent laie, gent clergie ${ }^{11}$.

13 En partant de l'importance du terme chevalerie dans le système hiérarchique de la société médiévale, Gerson propose l'alignement sur un suffixe nominal -ie. Dans cinq sermons différents ${ }^{12}$, datables de 1397 à 1413 , il présente les trois états par les termes : clergie, chevalerie, bourgeoisie.

Selon le système ternaire de Gerson, « ceux qui travaillent » seraient les représentants de la bourgeoisie. La société, dans son ensemble et dans ses parties, est censée tirer le plus grand profit du schéma tri-fonctionnel, selon lequel chaque ordre se voit impartir une mission. En fait, cette conception d'origine religieuse est plus un mythe qu'une réalité. Elle rend mal compte de la diversité qu'il y a dans chacun des ordres, des conflits qui surgissent en leur sein ou entre eux. Le concept « ordre » a une valeur bien plus grande et une plus vaste portée. Pour Johan Huizinga, chaque groupement, chaque fonction, chaque métier devient un "ordre", si bien que, "à côté de la division de la société en trois ordres, il peut s'en présenter une en douze $»^{13}$. L'auteur de L'automne du Moyen Age conclut que les mots «estat " et "ordre" s'appliquent, au Moyen Age, à un grand nombre de groupements humains qui nous semblent très dissemblables: «les états du royaume, mais aussi les métiers, l'état de mariage et celui de virginité, l'état de péché, les quatre 'estats de corps et de bouche "à la cour : panetiers, échansons, officiers tranchants et 
maîtres-queux, les ordres cléricaux: prêtres, diacres, sous-diacres, etc., les ordres monastiques, les ordres chevaleresques $»^{14}$.

A la fin du $11^{\mathrm{e}}$ siècle, les divers métiers spécialisés apparaissent. Le mot bourgeois fait son apparition. Or, lentement, mais continuellement, la société change. La réalité sociale n'a jamais correspondu à sa division théorique en trois ordres. L'apparition de nouveaux termes à l'intérieur du schéma ternaire rend compte de cette évolution. Cette tripartition n'explique plus rien, quand l'essor économique et urbain fait surgir les bourgeois, dont il est bien artificiel, selon Pierre-Yves Badel ${ }^{15}$, de faire des « laboureurs ». L'enrichissement des marchands et des banquiers est d'autant plus troublant qu'ils ne font pas ce qu'on appelle proprement « labourer ».

Quant à l'expression linguistique de cette réalité sociale, il faut pourtant reconnaître que Gerson est resté isolé dans sa tentative pour fixer l'ordre social sur la tonalité en « $i$ majeur ». Jean Batany explique que "pour les Français du $15^{\mathrm{e}}$ siècle, le suffixe -ie était sans doute à la fois trop peu étoffé pour fournir une base sérieuse à l'établissement autoritaire d'une idéologie, et trop abstrait pour permettre de classer les gens au niveau de leur rôle social $»^{16}$.

17 Une formulation idéologique moins abstraite, mais aussi rationnelle que celle de Gerson aurait pu alors s'établir sur les suffixes d'agent -ier et -eur, attestés dans notre premier série d'exemples, relevés dans les ballades d'Eustache Deschamps, où ces suffixes joints à la base constituent la désignation d'un métier concret, exercé par une personne représentant cette catégorie socio-professionnelle concrète. L'absence ou le caractère abstrait d'un suffixe commun aux constituants du schéma ternaire, décrivant les structures sociales fondamentales de la société, s'oppose donc à la productivité du suffixe d'agent ( -ier, -eur, -ien ) sur le plan fonctionnel, c'est-à-dire pour la désignation des catégories socio-professionnelles.

\section{Les « estats » féminins}

Dans un deuxième temps, nous nous intéresserons à un autre aspect lié au vocabulaire social : l'expression du genre féminin pour les « estats » de la société médiévale.

Dans ce grand système des « estats » du monde médiéval, comment le marquage du genre féminin s'effectue-t-il ? Comment la langue enregistre-t-elle cette réalité humaine et sociale?

20 Les exemples cités jusqu'ici confirment le concept de la domination grammaticale des mâles. En effet, le schéma triparti qui domine les conceptions que l'on se fait de la société chrétienne n'accorde aucune place spécifique aux femmes. Il hiérarchise «ordres» ou " conditions » - chevaliers, clercs et vilains -, mais cette pyramide, où ceux qui prient et ceux qui combattent ou rendent justice rivalisent à qui occupera la première place, ne prévoit pas de « condition féminine ».

21 En ancien français, le masculin, genre non-marqué, a droit aux indices de «cas». Le féminin, déjà marqué, n'y a généralement pas droit ${ }^{17}$. La dame peut donc être, dans un texte du $12^{\mathrm{e}}$ siècle, sujet ou objet du procès sans distinction morphologique, en face de li chevaliers - le chevalier, substantif masculin de la première déclinaison caractérisée par la présence d'un -s de flexion au cas sujet singulier, ou de li sire - le seigneur, opposés par une altérité radicale. 

$12^{\mathrm{e}}$ au $15^{\mathrm{e}}$ siècle ", Jean Batany constate que c'est seulement au $15^{\mathrm{e}}$ siècle qu'apparaît, dans certaines oeuvres, un catalogue systématique des femmes rangées par fonction sociale (ou par accompagnement matrimonial d'une fonction masculine) ${ }^{20}$. A l'écrasement de la diversité dans le domaine grammatical répond la faiblesse de la valeur sociale de la femme. Son statut social semble défini à peu près uniquement par sa féminité. Dans la vie pratique, il n'y avait pas une opposition fonctionnelle aussi nette entre la femme du seigneur et celle du vilain qu'entre leurs maris. La femme s'est trouvée définie avant tout par son corps, son sexe et ses relations avec des groupes familiaux, c'est-à-dire par son rôle d'épouse et de mère. C'est l'appartenance à un certain niveau social qui est à la base des différents types dans l'image du sexe féminin au Moyen Age. La notion de fonction ne s'avère que peu pertinente étant donné que, près de n'importe quel homme, la femme fait à peu près la même chose.

Quant au reflet linguistique de cette réalité naturelle et humaine, on constate que la marque morphologique du féminin en -e (dame, reine, fille, pucelle ...) est celle de l'adjectif de la première classe (bonne, belle ... ). L'adjectif référant à une réalité inanimée, la qualité, l'opposition de genre de l'adjectif pouvait donc être perçue comme une opposition valable au niveau des choses. C'est pourquoi, selon Batany, la marque morphologique du féminin en -e paraît caractéristique d'un niveau un peu méprisé ${ }^{21}$. Rappelons également l'importance du critère phonétique selon lequel l'expression de la marque du féminin -e se réduisait de plus en plus à une résonance post-accentuelle affaiblie.

Les rangs supérieurs de la société ne pouvaient se contenter de la diminution de leur prestige social à travers l'utilisation du système de marque de genre employé pour les qualités. En effet, les représentantes des couches sociales élevées vont marquer grammaticalement leur féminité à l'aide d'un suffixe qui, en même temps, indique l'appartenance aux rangs supérieurs de la société : il s'agit du suffixe-esse dont le double fonctionnement (marquage du genre féminin, appartenance à un niveau social supérieur), se reflète dans les désignations princesse, comtesse, duchesse, baronesse. Le sexe féminin est en quête de la reconnaissance de sa dignité et de sa valeur à travers l'utilisation d'un suffixe spécifique qui semble être réservé essentiellement aux classes supérieures de l'humanité. La dignité que représente le suffixe -esse apparait également dans des désignations féminines mettant en avant l'aspect fonctionnel, comme par exemple dans le terme abesse, attesté depuis le $12^{\mathrm{e}}$ siècle. Dans le Dit des Cordeliers de 
Rutebeuf, nous relevons deux occurrences de ce terme désignant la femme responsable de la gestion d'une abbaye :

L'abeasse qui cloche la cloiche dou clochier

Fist (le pape) devant li venir, qu'i la vëist clochier. (...)

Or les (les Cordeliers) veut l'abeesse de la vile banir. ${ }^{22}$

Dans la première moitié du $14^{\mathrm{e}}$ siècle, apparaît dans le Mireuer du Monde ${ }^{23}$ derrière le chevalier une chevaleresse. Ce féminin est également attesté, vers la même époque, chez Watriquet. Sa diffusion témoigne de l'échec de la concurrente chevalière, choisi encore au siècle précédent par Jean de Meun afin de désigner la représentante féminine de la chevalerie dans le défilé des femmes confessées par Faux-Semblant :

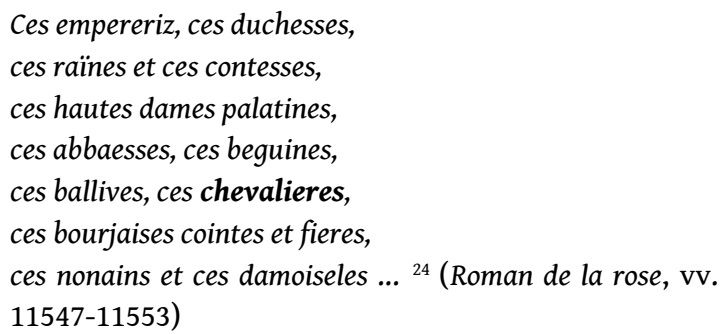

Sur les douze désignations féminines de cet extrait, Jean de Meun n'emploie que quatre formations simples en -e sur le masculin: baillives (masculin : bailli / baillif), chevalieres (masculin : chevalier), bourjaises (masculin : borjois), damoiseles (masculin : damoisel) ${ }^{25} . \mathrm{Si}$ nous comparons cette série de formes en -e aux autres formes( suffixe -esse: duchesses, contesses, abbaesses; féminin en -iz: empereriz), nous constatons qu'il s'agit, en somme, des grades les moins élevés de l'énumération des femmes de ce défilé.

Il faut attendre l'œuvre d'une poétesse du début du $15^{\mathrm{e}}$ siècle avant de trouver dans un texte une revue systématique des estats féminins, entièrement autonome et non plus en complément des estats masculins : il s'agit du Livre des trois vertus (1405) de Christine de Pizan. Mais le vocabulaire qu'on y trouve ne reflète pas du tout la richesse morphologique des formations de féminin, il est plutôt pauvre et discret. De l'analyse de Jean Batany résulte que toute la première partie, consacrée à la très haute noblesse, emploie à peu près uniquement le mot princesse; dans la seconde, la petite noblesse semble divisée en deux catégories, les dames et damoiselles de court, ou femmes de court, et les dames baronnesses qui résident aux châteaux; pour le reste de la société, on a surtout des périphrases avec femme ou dame: dames de religion, femmes d'estat des bonnes villes et bourgoises, femmes des mestiers (" mariées aux hommes des mestiers »), simples femmes de labour ${ }^{26}$. Dans l'ensemble, Christine de Pizan nous présente donc essentiellement des épouses dont l'estat est défini par celui de leur mari. Nous rencontrons cette image du statut de la femme comme résultant de la position sociale de son mari, de nouveau dans le Quadrilogue invectif (1422) d'Alain Chartier :

... quant un varlet cousturier et la femme d'un homme de bas estat osent

porter l'abit d'ung vaillant chevalier et d'une noble dame... ${ }^{27}$

Dans cet exemple du Quadrilogue invectif, Alain Chartier attribue au représentant masculin des laboratores une fonction précise, celle du varlet cousturier, le statut de la femme, au même niveau social, est défini par rapport à celui de son mari. La noble dame, quant à elle, puise sa raison d'être dans son appartenance à la noblesse. Le critère de la fonction est donc absent de l'image de la femme. Il n'est que peu présent dans le Livre des trois vertus de Christine de Pizan, où les métiers spécifiquement féminins sont peu signalés, sauf les 
servantes et les chambrières - «catégorie socio-professionnelle » très fréquente dans les textes du Moyen Age ${ }^{28}$.

Dans la série de désignations féminines développée par Christine de Pizan, nous ne relevons que quatre formations de féminin par dérivation du masculin correspondant: princesses, baronnesses, damoiselles, bourgeoises. La haute noblesse, celle qui porte des titres, confisque le suffixe -esse et la petite noblesse le radical dam- (dames, damoiselles). En effet, le féminin damoiselle, désignant en ancien français une jeune fille de rang social élevé, est employé en moyen français pour une femme de petite noblesse ou pour une femme noble mariée à un bourgeois ${ }^{29}$. Les exemples nous montrent que la hiérarchie entre les suffixes esse (princesse) et -e (bourgeoise) subsiste. Elle se confirme dans un autre texte du début du $16^{\mathrm{e}}$ siècle : les Souhaitz des femmes qui répondent aux Souhaitz des hommes ${ }^{30}$. Les résultats de l'analyse de Jean Batany montrent que parmi les 29 désignations féminines dans les Souhaitz des femmes, onze ont leur correspondant chez les hommes, les quatre premières la royne, la duchesse, la contesse, la chevaleresse - et sept autres : la borgoise, la marchande, la présidente, l'advocade, la maquerelle, la coquine et la bergère ${ }^{31}$. Nous retrouvons donc la même répartition: le haut niveau social est caractérisé par l'utilisation du suffixe -esse (s'y ajoute une autre forme à morphologie originale : royne), tandis que les formes féminines en - $e$ désignent des représentantes des niveaux sociaux inférieurs. Comme nous avons pu le constater, le terme chevaleresse est également attesté dans ce texte où il répond à l'utilisation de la variante masculine dans les Souhaitz des hommes. Mais ce type de fonctionnement ne se répète pas pour le laboureur, occurrence relevée chez les hommes, et la *laboureresse. Cette forme, morphologiquement attendue, n'est pas attestée.

Il est important de signaler que dans ce texte, les Souhaitz des femmes, apparaissent pour la première fois dans le cadre de la présentation des «estats " féminins, d'autres métiers proprement féminins, spécifiant l'image de la féminité au Moyen Age : la mesnagière, la lavandière (femme qui lave le linge à la main), la filandière (femme qui fait le métier de filer).

Bien sûr, nous ne trouvons pas dans tous les textes de la littérature médiévale des reflets aussi détaillés et spécifiques du statut de la femme et de son activité au sein de la société. Dans beaucoup d'œuvres, les désignations masculines pour les représentants des « estats » $\mathrm{du}$ monde sont dominantes. Le féminin cohabite souvent avec son correspondant masculin:

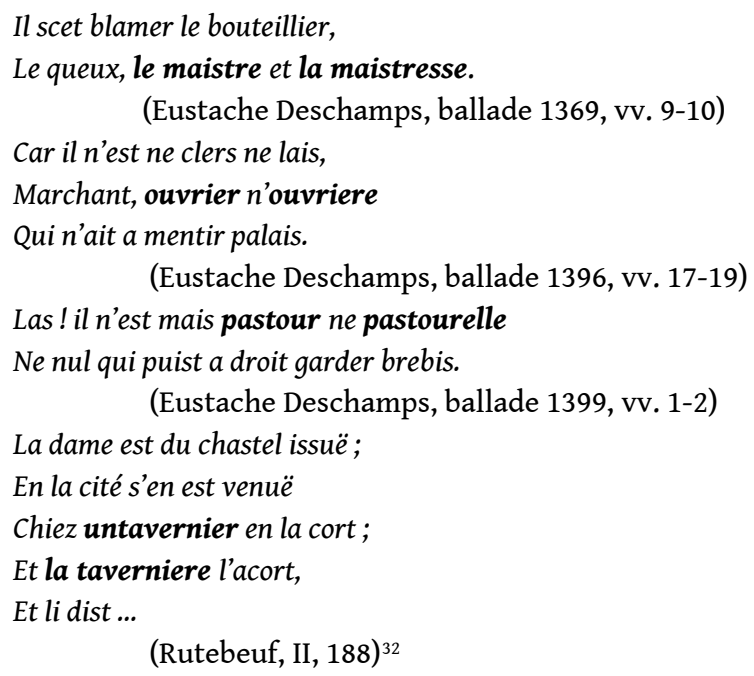


Moyen Age ont longtemps conçu « la femme » comme une «catégorie $»^{38}$ faisant partie de la vie en société. Mais ce n'est que tardivement qu'ils y ont fait intervenir distinctions sociales et activités professionnelles. Avant d'être paysanne, châtelaine ou chirurgienne, la femme s'est trouvée définie, en premier lieu, par son corps, son sexe, et ses relations avec des groupes familiaux. C'est par rapport à un homme ou à un groupe d'hommes qu'on a déterminé sa personnalité juridique. Les textes étudiés nous font ressentir la difficulté liée à la détermination du statut de la femme qui, très souvent, n'est que « la femme de » (la femme du chevalier, la femme de l'escuier) avant de s'imposer, au niveau social et linguistique, en tant qu'individu doté d'une personnalité juridique, morale et économique.

Avant d'exercer une activité sociale précise, un métier «typiquement féminin » pour ce contexte médiéval, la femme se caractérise surtout par son appartenance à un certain 
niveau social, défini par celui de son mari : les femmes des mestiers sont, comme le précise Christine de Pizan dans son Livre des trois vertus, des femmes mariées aux hommes des mestiers, qui représentent " ceux qui travaillent » selon le schéma tri-fonctionnel. Comme les exemples attestés le confirment, la langue médiévale a une préférence pour des suffixations différentes selon la position sociale de la femme : -esse pour le haut niveau social et $-e$ de type adjectival pour les niveaux inférieurs. Morphologie et sociologie fonctionnent en parallèle.

Selon nous, la mise en relation de critères linguistiques et de facteurs socio-historiques ne peut pas être négligée dans une analyse consacrée au vocabulaire social. Cette approche nous permet de comprendre pourquoi dans cette société dominée par les mâles, la voix féminine ne déploie pas toutes ses tonalités.

\section{NOTES}

1. Toutes ces occurrences sont attestées au volume V des Oeuvres Complètes d'Eustache Deschamps, Paris, 1887.

2. Dans son article consacré aux " estats du monde " chez Eustache Deschamps, Jean Batany montre que ce « catalogue des métiers ne s'oriente ni dans le sens d'une hiérarchie, ni dans celui d'une vision simple limitée à un petit nombre de composants; il constitue une structure polymère ouverte, dont l'ordre et la nomenclature sont laissés à l'arbitraire de l'auteur, ou à ses rencontres quotidiennes ». (1999, Actes du Colloque du Centre d'Etudes Médiévales de l'Université de Picardie, Autour d'Eustache Deschamps, Amiens, p. 2)

3. Jacques Le Goff, 1989, L'homme médiéval, édition française, p. 17.

4. Georges Dumézil, 1958, L'Idéologie tripartie des Indo-Européens, Collection Latomus.

5. Voir, par exemple, Georges Duby, 1979, Les trois ordres ou l'imaginaire du féodalisme, Paris, et 1982, "A propos des trois ordres» (Esquisses 21-25), in Apollon sonore et autres essais: vingt-cinq esquisses de mythologie, Paris, pp. 205-253. Jacques Le Goff, 1964, Civilisation de l'Occident médiéval, Paris, et 1971, Le Moyen Age, Paris.

6. Jean Batany, 1992, Approches langagières de la société médiévale, Caen, Paradigme, p. 203.

7. Ibid., pp. 203-204.

8. A partir du $11^{\mathrm{e}}$ siècle, les chevaliers fusionnent avec l'ancienne noblesse carolingienne car ils partagent les mêmes privilèges : guerre, loisirs, pouvoir de commandement sur les paysans, et surtout la conscience de se rattacher, par la généalogie, à un ancêtre divin ou héroïque, fondateur par excellence de la maison noble. Ainsi s'est constituée ce que l'on appelle la noblesse médiévale qui transmet sa condition par le sang. (Voir article de Robert Delort «Ceux qui combattent : les chevaliers ", in La vie au Moyen Age, Paris, Seuil, 1982, pp. 160-186).

9. Ed. Robert Puchell, Berlin et Paris 1881, pp. 98-270 ; cité par Jean Batany, op. cit., p. 205.

10. Oeuvres complètes de Rutebeuf, éd. E. Faral et J. Bastin, t. 1, pp. 377-381.

11. Dans la complainte de Guillaume de Saint-Amour, on rencontre un autre syntagme de cette structure : la gent Justinien (Poèmes de l'infortune et autres poèmes, 1986, p. 222). Rutebeuf se sert de cette expression afin de désigner les étudiants de la faculté de droit.

12. Gerson, Oeuvres complètes, éd. Mgr Glorieux, vol. VII, $2^{\mathrm{e}}$ partie, Paris, 1968, pp. 547, 753, 910, 1013 et 1151 ; cité par Jean Batany, Approches langagières de la société médiévale, p. 205.

13. Johan Huizinga, 1975, L'automne du Moyen Age, (édition française) éd. Payot, p. 85. 
14. Ibid., pp. 85-86.

15. Pierre-Yves Badel, 1984, Introduction à la vie littéraire du Moyen Age, Paris, p. 19 («L'ordre et l'histoire »).

16. Jean Batany, op. cit., p. 205.

17. Même les déclinaisons dites "asymétriques », caractérisées par la présence d'un «-s » de flexion au cas sujet singulier (singulier, cas sujet : la flors, cas régime : la flor, pluriel : les flors), ou les représentants du troisième modèle de déclinaison à alternance radicale (la suer / la seror - les serors) subissent un alignement analogique sur le type majoritaire de la première déclinaison : la dame (singulier) - les dames (pluriel).

18. Rutebeuf, Poèmes de l'infortune et autres poèmes, 1986, pp. 166, 176, 178, 194, 196, 200, 204, 210, 216, 226, 230, 236, 238, 262, 266.

19. Ibid., pp. 42, 62, 84, 214.

20. Jean Batany, 1992, op. cit., p. 192.

21. Ibid., pp. 193-194.

22. Rutebeuf, 1986, op. cit., p. 200.

23. Ed. par Méon, Les vers sur la mort de Thibaud de Marly..., Paris, 1835, pp. 75-79, cité par Jean Batany, op. cit., p. 195.

24. Roman de la Rose, éd. Lecoy, Champion 1966, tome II p. 102, cité par Jean Batany, 1992, op. cit., p. 195.

25. Le masculin damoisel désigne le jeune homme noble qui n'est pas encore reçu chevalier. La forme féminine correspondante est utilisée en ancien français pour les jeunes filles de rang social élevé, mais elle s'applique également au statut de servante. En moyen français, ce lexème subit un renforcement du trait de «noblesse » au détriment de ceux de « jeunesse » et de " célibat ». (Nelly Andrieux-Reix, 1991, Ancien Français. Fiches de vocabulaire, $3^{\mathrm{e}}$ édition, PUF, pp. 227-232).

26. Selon Jean Batany, 1992, op. cit., p. 196.

27. Alain Chartier, Le Quadrilogue invectif, Droz, $2^{\mathrm{e}}$ éd., 1950, p. 41.

28. La pauvreté du vavasseur, hôte par excellence du chevalier errant dans les romans de Chrétien de Troyes, se manifeste à travers l'absence d'une servante ou chambrière dans sa maison : Li vavasors sergent n'avoit, for un tot seul qui le servoit, ne chanberiere ne meschine; (Chrétien de Troyes, Erec et Enide, publié par M. Roques, Champion, 1981, vv. 485-487).

Dans les ballades d'Eustache Deschamps, nous relevons plusieurs occurrences du terme " chambrière ", par exemple : ballade 1392, v. 6 : Car sires est et je suis chamberiere; ballade 1397, vv. 1-2: L'en treuve es droiz de Champaigne, ce tiens, Que la Brie est sa serve et sa chambriere; ballade 1426, v. 2 : Jehanne no chamberiere ...

29. Nelly Andrieux-Reix, op. cit., p. 231.

30. Il s'agit d'un petit poème prêtant un voeu à chaque type social.

Les souhaits des femmes, dans Montaiglon et Rothschild, Recueil de poésies françoises ..., tome III, 1856, pp. 147-153 ; cité par J. Batany, Approches langagières de la société médiévale, p. 197.

31. Selon Jean Batany, 1992, op. cit., p. 197.

32. Oeuvres complètes de Rutebeuf, éd. Faral / Bastin, tome II.

33. Eustache Deschamps, Oeuvres complètes, V, p. 85, ballade 1397, v. 26.

34. Charles d'Orléans, Poésies, I, éd. par P. Champion, 1982, p. 44, ballade 25, vv. 22-23.

35. La Dance des femmes, de Martial d'Auvergne en 1486 (éd. par Luise Götz, 1934, Zeitschrift für französische Sprache und Literatur, LVIII, pp. 318-334) ; cité par J. Batany, 1992, op. cit., pp. 196-197.

36. Voir L'homme médiéval, sous la direction de Jacques Le Goff, Seuil, 1989, article «L'intellectuel » de Mariateresa Fumagalli Beonio Brocchieri, pp. 201-232.

37. Rutebeuf, 1986, op. cit., pp. 102-103.

38. Selon Christiane Klapisch-Zuber, 1989, « Les femmes et la famille », in L’homme médiéval, sous la direction de Jacques Le Goff, Seuil, p. 315. 


\section{AUTEUR}

\section{SABINE LEHMANN}

Ecole doctorale, Université de Paris 10 - Nanterre 